\title{
Short course of systemic corticosteroids in wheezy children: still an open question
}

\begin{abstract}
Introduction: We performed a real-life clinical study to identify the main indications for the prescription of short-course treatment with systemic glucocorticosteroids (GCS) for steroid naive children with acute virus-induced wheezing as well as to analyze the influence of such treatment on patients' serum cortisol level, other blood tests results and the length of stay in the hospital. Material and methods: The data of 44 patients who had acute wheezing, had no bacterial infection and were otherwise healthy were analyzed: 26 children received treatment with GCS and 18 children did not. Full blood count, biochemistry tests (Na, K, glucose) and blood cortisol levels of all patients were analyzed during treatment.

Results: The main indications for the short-term administration of systemic GCS were increased work of breathing, recurrent wheezing, clinical signs of atopy and a family history of asthma. Systemic GCS increased a sodium concentration $(p=0.014)$, decreased a cortisol level $(p=0.038)$, leukocyte $(p=0.043)$, neutrophil $(p=0.045)$, and eosinophil $(p<0.001)$ count in blood serum. The major reduction in the eosinophil count was observed in allergic children $(p=0.023)$. Older age was a risk factor for cortisol suppression $(p=0.018)$. The average length of stay in the hospital was longer in the intervention group $(p=0.039)$.

Conclusion: Even short-course treatment with systemic GCS decreases the serum cortisol level and has a significant effect on other blood tests results. Systemic GCS used for acute virus-induced wheezing treatment did not prove to reduce the average length of stay in the hospital. Objective criteria for initiation of such treatment are still lacking, which might consequently lead to the overuse of corticosteroids.
\end{abstract}

Key words: cortisol suppression, glucocorticosteroids, viral-induced wheezing, wheezing

Adv Respir Med. 2019; 87: 209-216

\section{Introduction}

Glucocorticosteroids (GCS) are immunosuppressive and anti-inflammatory agents that have been widely used in many pediatric conditions for more than 60 years [1]. Respiratory diseases are not an exception. Acute airway obstruction is the most common indication for short-course treatment with systemic GCS [1, 2].

Current evidence recommends systemic GCS as an effective and safe choice for diminishing symptoms of asthma exacerbation and croup, but in clinical practice, these drugs are used more widely. However, their role in other respiratory conditions is controversial $[1,2]$. To date, it remains unclear which children may benefit the most from systemic GCS and for whom the treatment is overused. Moreover, there is little evidence about the possible side effects of systemic GCS when they are given as short-course treatment for virus-induced wheezing in children [2, 3].

GCS can cause Cushing's syndrome, osteoporosis, arterial hypertension, growth failure, and obesity when they are used for a long time $[3,4]$. The main undesirable effects occur regarding suppression of the hypothalamic-pituitary-adrenal (HPA) axis and immune system [3, 4]. Electrolyte changes, hyperglycemia, cortisol suppression, and changes in circulating white cells are mostly analyzed in the adult population [4]. Their effects on children are still to be clarified.

Therefore, our aim was to perform a real-life clinical study to identify the main indications for prescription of short-course treatment with systemic GCS for steroid naive children with acute virus-induced wheezing as well as to analyze the influence of such treatment on patients' serum cortisol level, blood cells count, biochemistry

Address for correspondence: Valdone Miseviciene, Department of Pediatrics, Lithuanian University of Health Sciences Hospital, Eiveniu g. 2, LT-50161, Kaunas, Lithuania; e-mail: valdone.miseviciene@kaunoklinikos.It

DOI: 10.5603/ARM.a2019.0035

Received: 07.03.2019

Copyright (C) 2019 PTChP

ISSN 2451-4934 
results and the average length of stay in the hospital. We hope that our results can provide a background for more extensive and worldwide investigations in this field.

\section{Materials and methods}

This prospective real-life clinical study was performed between February 2016 and March 2017 in the Pediatric Department of Lithuanian University of Health Science Hospital (LUHSH) Kaunas Clinics. Approval from the institutional ethics committee (approval No. BEC-MF-137, December 15, 2015) was obtained. Parents/carers were provided with detailed information sheets and discussed the procedures with the researcher prior to supplying their written consent form.

Children (1 month to 18 years old) who had acute wheezing, had neither bacterial infection nor other diseases, were included in the study. No patient had previously received systemic GCS for any reason. On admittance to the hospital, the clinic staff was informed about required investigations and evaluation of the patient's clinical signs, but no specific recommendations for treatment were given to the physicians.

Age, clinical signs, family history, anamnesis about previous wheezing episodes and atopy, as well as prescribed treatment, full blood count, biochemistry tests ( $\mathrm{Na}, \mathrm{K}$, glucose), and blood cortisol levels of all patients were analyzed. Respiratory rate was evaluated according to the age of patients. Blood tests were performed on the first, second, and fourth days of treatment. On the first day of admittance, blood was drawn at any time before prescribed treatment, and on the other days, blood was drawn at 9 a.m. Systemic GCS were given routinely and immediately after the collection of blood tests. The results between the first and the second day and between the first and the fourth day were evaluated and compa- red. Finally, we analyzed two groups of patients: those who received short-course treatment with GCS (intervention group) and those who did not (control group).

All blood samples were examined in the laboratory of LUHSH. Blood cell count was analyzed using an automatic hematologic analyzer (Sysmex XE-5000). Fluorescence flow cytometric analysis was performed to evaluate white blood cell counts and their differentials. An impedance method was used to calculate the platelet count. In some cases, the platelet count was recalculated using fluorescent reagents. A glucose level and sodium and potassium concentration were analyzed by the SYNCHRON system. The potentiometric titration method was used to evaluate the concentration of electrolytes. The glucose level was estimated with the oxygen rate method employing a Beckman Coulter Oxygen electrode. Cortisol test was analyzed by ST AIA-PACK CORT. Competitive enzyme immunoassay method was used to evaluate a cortisol concentration in the blood plasma.

Evaluation of the cortisol level during treatment was performed according to reference values of the cortisol concentration for all age groups (177-578 nmol/L in the morning, $<434 \mathrm{nmol} / \mathrm{L}$ in the afternoon). The change in the cortisol level was set after estimation of how elevated, normal, or decreased the cortisol values were during treatment (Table 1). Because of the wide range of normal cortisol values, the circadian rhythm of the cortisol concentration, and the influence of possible increasing cortisol concentration factors, we did not use the exact cortisol concentration in the statistical analysis.

Statistical analysis was performed using SPSS software (version 23.0). Statistical significance was set at $p<0.05$. Correlation coefficients were used to measure the statistical strength of the correlation between two variables. Spear-

Table 1. Changes in cortisol level during treatment

\begin{tabular}{ll}
\hline Level & Evaluation of the change of cortisol \\
\hline 2 & $\begin{array}{l}\text { The cortisol concentration was decreased. It increased and exceeded normal values in the blood plasma after treat- } \\
\text { ment }\end{array}$ \\
1 & The cortisol concentration was decreased in the blood plasma and became normal after treatment/the cortisol concen- \\
tration was normal in the blood plasma and increased after treatment \\
0 & The cortisol concentration maintained the same blood plasma level after treatment \\
-1 & The cortisol concentration was normal in the blood plasma and decreased after treatment/the cortisol concentration \\
-2 & was increased in the blood plasma and became normal after treatment \\
& The cortisol concentration was increased in the blood plasma and decreased after treatment
\end{tabular}


Table 2. Comparison of intervention and control groups

\begin{tabular}{|c|c|c|c|c|c|c|c|c|}
\hline \multicolumn{2}{|l|}{ Variable } & \multicolumn{4}{|c|}{ Intervention group $(n=26)$} & \multicolumn{2}{|c|}{ Control group $(n=18)$} & $P$ value \\
\hline \multicolumn{2}{|l|}{ Gender } & \multicolumn{4}{|c|}{$8(30.8 \%)$ female; $18(69.2 \%)$ male } & \multicolumn{2}{|c|}{$\begin{array}{c}10(55.6 \%) \text { female } \\
8(44.4 \%) \text { male }\end{array}$} & 0.091 \\
\hline \multicolumn{2}{|l|}{ Average age } & \multicolumn{4}{|c|}{3.4} & \multicolumn{2}{|c|}{1.8} & 0.08 \\
\hline \multicolumn{2}{|l|}{ Age group } & \multicolumn{4}{|c|}{$\begin{array}{c}10(38.5 \%)<2 y \\
11(42.3 \%) 2-5 y \\
5(19.2 \%)>5 y\end{array}$} & \multicolumn{2}{|c|}{$\begin{array}{c}11(61.11 \%)<2 y \\
6(33.3 \%) 2-5 y \\
1(5.56 \%)>5 y\end{array}$} & 0.243 \\
\hline \multicolumn{2}{|c|}{ Recurrent wheezing } & \multicolumn{4}{|c|}{$19(73.08 \%)$} & \multicolumn{2}{|c|}{$1(5.56 \%)$} & $<0.001$ \\
\hline \multicolumn{2}{|c|}{ Signs of atopy } & \multicolumn{4}{|c|}{$19(73.08 \%)$} & \multicolumn{2}{|c|}{$2(11.1 \%)$} & 0.001 \\
\hline \multicolumn{2}{|c|}{ Usage of accessory muscles } & \multicolumn{4}{|c|}{$15(57.69 \%)$} & \multicolumn{2}{|c|}{$1(5.56 \%)$} & 0.007 \\
\hline \multicolumn{2}{|c|}{ Increased respiratory rate } & \multicolumn{4}{|c|}{$19(73.08 \%)$} & \multicolumn{2}{|c|}{$7(38.89 \%)$} & 0.160 \\
\hline \multicolumn{2}{|c|}{ Desaturation $<92 \%$} & \multicolumn{4}{|c|}{$8(30.77 \%)$} & \multicolumn{2}{|c|}{$5(27.77 \%)$} & 0.552 \\
\hline \multicolumn{2}{|c|}{ Family history of asthma } & \multicolumn{4}{|c|}{$8(30.11 \%)$} & \multicolumn{2}{|c|}{0} & 0.044 \\
\hline \multirow[t]{2}{*}{ Leukocytes } & $\begin{array}{c}\text { Average value before } \\
\text { treatment }\end{array}$ & & 13.29 & $10^{9} / \mathrm{L}$ & & 10.26 & $10^{9} / \mathrm{L}$ & 0.083 \\
\hline & First day of treatment & & -1.5 & $10^{9} / \mathrm{L}$ & & $1.22>$ & $10^{9} / \mathrm{L}$ & 0.043 \\
\hline Neutrophils & Fourth day of treatment & & -4.27 & $10^{9} / \mathrm{L}$ & & $0.14>$ & $10^{9} / \mathrm{L}$ & 0.045 \\
\hline Eosinophils & First day of treatment & & -0.17 & $10^{9} / \mathrm{L}$ & & 0.019 & $10^{9} / \mathrm{L}$ & $<0.001$ \\
\hline & & Atopic c & hildren & Non-atop & children & & & \\
\hline & & $-0.18>$ & $10^{9} / \mathrm{L}$ & -0.07 & $10^{9} / \mathrm{L}$ & & & \\
\hline Eosinophils & Fourth day of treatment & & -0.15 & $10^{9} / \mathrm{L}$ & & $0.05>$ & $10^{9} / \mathrm{L}$ & 0.007 \\
\hline & & Atopic c & hildren & Non-atop & children & & & \\
\hline & & $-0.17>$ & $10^{9} / \mathrm{L}$ & -0.003 & $\times 10^{9} / \mathrm{L}$ & & & \\
\hline Lymphocytes & First day of treatment & & $0.2 \times$ & $10^{9} / \mathrm{L}$ & & $1 \times$ & $10^{9} / \mathrm{L}$ & 0.257 \\
\hline Monocytes & First day of treatment & & 0.03 & $10^{9} / \mathrm{L}$ & & $1.5 \times$ & $10^{9} / \mathrm{L}$ & 0.863 \\
\hline Platelets & First day of treatment & & 34.5 & $10^{9} / \mathrm{L}$ & & $15.9>$ & $10^{9} / \mathrm{L}$ & 0.322 \\
\hline Sodium & First day of treatment & & 1.69 & $\mathrm{~mol} / \mathrm{L}$ & & $0.18 \mathrm{r}$ & $\mathrm{mol} / \mathrm{L}$ & 0.014 \\
\hline Potassium & Fourth day of treatment & & 0.25 & $\mathrm{~mol} / \mathrm{L}$ & & -0.08 & $\mathrm{nmol} / \mathrm{L}$ & 0.009 \\
\hline Glucose & First day of treatment & & -0.66 & $\mathrm{nmol} / \mathrm{L}$ & & -0.25 & $\mathrm{nmol} / \mathrm{L}$ & 0.653 \\
\hline Cortisol level & First day of treatment & $\begin{array}{l}\text { Maintain } \\
\text { in the } \\
\text { same level }\end{array}$ & $\begin{array}{c}\text { Decreased } \\
\text { by one } \\
\text { level }\end{array}$ & $\begin{array}{c}\text { Decreased } \\
\text { by two } \\
\text { levels } \\
\end{array}$ & $\begin{array}{c}\text { Increased } \\
\text { by one } \\
\text { level }\end{array}$ & $\begin{array}{l}\text { Maintain } \\
\text { in the } \\
\text { same level }\end{array}$ & $\begin{array}{c}\text { Decreased } \\
\text { by one } \\
\text { level }\end{array}$ & \\
\hline & & $\begin{array}{c}11 \\
(42.31 \%)\end{array}$ & $\begin{array}{c}9 \\
(34.61 \%)\end{array}$ & $\begin{array}{c}5 \\
(19.23 \%)\end{array}$ & $\begin{array}{c}1 \\
(3.85 \%)\end{array}$ & $\begin{array}{c}15 \\
(83.34 \%)\end{array}$ & $\begin{array}{c}3 \\
(16.66 \%)\end{array}$ & $\begin{array}{c}\mathbf{0 . 0 3 8} \\
(r=0.252)\end{array}$ \\
\hline
\end{tabular}

man's rank correlation coefficient was used for nonparametric variables. Pearson correlation coefficient was used for parametric variables. The Mann-Whitney test and analysis of variance were used for comparison of the change in rates between the intervention group and the control group. $\mathrm{Chi}^{2}$ test was used for qualitative variables.

\section{Results}

A total of 44 patients diagnosed with viral-induced wheezing were included in the stu- dy: 26 children in the intervention group and 18 children in the control group. There was no statistically significant difference in distribution by gender ( $p=0.091)$ or age $(p=0.243)$ between the groups (Table 2).

Methylprednisolone was applied for treatment in 15 (57.7\%) patients, prednisolone in $7(26.9 \%)$, and both drugs were used one after another in 4 (15.4\%) patients. An average equivalent dose of methylprednisolone was $1.2 \mathrm{mg} / \mathrm{kg}$ $(\min =0.4 \mathrm{mg} / \mathrm{kg}, \max =2 \mathrm{mg} / \mathrm{kg})$, and the average duration of administration was 4.4 days 


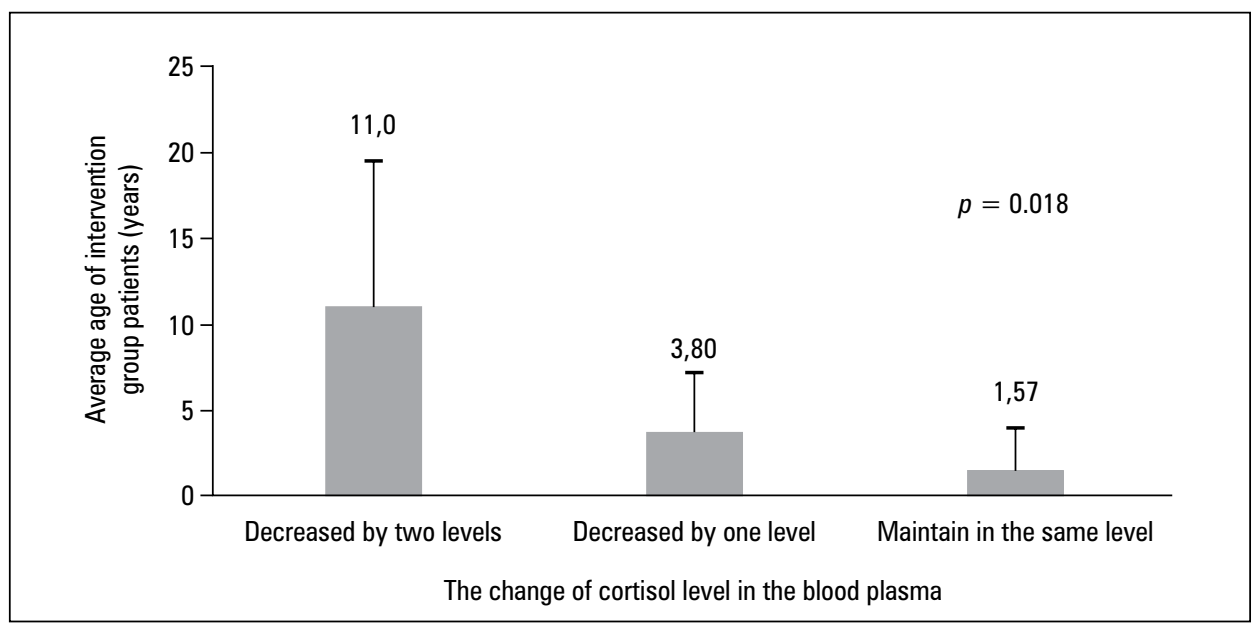

Figure 1. Average age of glucocorticosteroids users in the cortisol level groups after fourth day of treatment

( $\min =1$ day, $\max =15$ days). All patients in the study were treated with salbutamol inhalations and intravenous fluids (isotonic sodium chloride solution).

Recurrent wheezing, signs of atopy, a family history of asthma, and the use of the accessory muscles in breathing at rest had statistical significance in the intervention group. There was no statistically significant difference in increased respiratory rate or desaturation $<92 \%$ between the intervention and control groups (Table 2). There was no evidence of cyanosis in the study groups.

There was no statistically significant difference in the initial average count of leukocytes in the blood between the study groups before the treatment (Table 2).

The decrease in leukocytes and eosinophils after the first day of treatment and the decrease in neutrophils after the fourth day of treatment were statistically significant in the intervention group (Table 2).

Signs of atopy were observed in 19 (73.08\%) children in the intervention group, and 7 (26.92\%) patients were nonatopic. Atopic children had a major reduction in the eosinophil count after the first day of treatment in the intervention group $(p=0.023)$, and the same effect remained after the fourth day as well ( $p=0.048$; Table 2).

There was no statistically significant difference in changes in other blood cells, such as lymphocytes $(p=0.257)$, monocytes $(p=0.863)$, and platelets $(p=0.322)$ during treatment (Table 2).

The sodium concentration was significantly higher after the first day of treatment in the intervention group than in controls (Table 2). There was one case of hypernatremia $(147 \mathrm{mmol} / \mathrm{L})$ and one case of hypokalemia $(3.4 \mathrm{mmol} / \mathrm{L})$ after the first day of treatment in the intervention group. Abnormal changes in the electrolytes concentration were not observed in those without GCS treatment.

There was no statistically significant difference in changes in the blood plasma glucose level between the study groups (Table 2). Only one case of hyperglycemia $(15.64 \mathrm{mmol} / \mathrm{L})$ was registered after the first day of treatment in the GCS users group.

The cortisol concentration was maintained at the same level after the first day of treatment in $11(42.31 \%)$ cases, whereas it decreased by one level in 9 (34.62\%) patients and by two levels in 5 (19.23\%) individuals treated with systemic GCS. There was one distinctive case in the intervention group in which the cortisol level increased by one level after the first day of GCS treatment. In this particular case, the cortisol concentration was normal and became increased in the blood plasma after treatment. The cortisol concentration was maintained at the same level in 15 cases (83.33\%) after the first day of treatment in the control group and decreased by one level in $3(16.67 \%)$ cases; specifically, in all 3 of these cases, the cortisol concentration was increased in the blood plasma and became normal after the first day of treatment. There was a statistically significant difference in cortisol changes after the first day of treatment between the study groups $(r=0.252 ; p=0.038$; Table 2). The decrease in the cortisol level in those who were treated with GCS was statistically significant after the fourth day of treatment as well $(r=0.262 ; p=0.047)$. The average age of children was higher in the groups with a cortisol decrease ( $p=0.018)$ after the fourth day of using systemic GCS (Figure 1). The average length of 
stay in the hospital was significantly longer in the intervention group -6.26 days, compared to 4.17 in the control group ( $p=0.039$ ). There was no statistically significant difference in the average length of stay in the hospital between allergic (6.79 days) and non-allergic (4.86 days) children treated with systemic GCS ( $p=0.224$ ).

\section{Discussion}

The prevalence of asthma and other wheezing disorders in children is increasing worldwide [5, 6]. The proper diagnosis and best treatment in many pediatric cases is still an open question.

It is already established that half of the children wheeze at least once until the age of 6 years, but not all of them have or will have asthma, which is treated mainly with GCS $[7,8]$. However, many wheezy children receive GCS for a certain period of time, especially during exacerbations caused by viral respiratory infections [1, 7-9]. Most likely, some of these children undergo such treatment without need and confirmed efficacy [7, 10]. Moreover, possible side effects are usually not taken into account, because it is thought that short courses of GCS are relatively safe [7]. Therefore, our aim was to analyze the existing evidence in the literature and to compare these data with our findings in a real-life clinical trial. We focused on indications for prescription of systemic GCS in children with acute virus-induced wheezing as well as on the clinical effects of such treatment.

Both groups in our study were considered to be equal and comparable due to similar distribution by age and gender. We found that increased work of breathing at rest was the only significant clinical factor representing the severity of wheezing and was the main indication for systemic GCS prescription. The frequency of desaturation $\left(\mathrm{SpO}_{2}<92 \%\right)$ and respiratory rate were similar in both study groups, and the values were nearly normal. Recurrent wheezing, signs of atopy, and a family history of asthma were other significant factors found more often in the intervention group.

According to the latest recommendations, systemic GCS are not advised for bronchiolitis but are strongly recommended for children with acute asthma exacerbation $[6,11]$. Evidence supporting the benefits of GCS for viral-induced wheeze among preschool-aged children is very limited and conflicting [6, 9, 12]. Usually, such treatment is recommended and justified for children with severe wheezing and positive asthma predictive index (API), which includes a combination of recurrent wheezing and risk factors, such as parental history of asthma, personal history of atopic dermatitis, allergic sensitization to inhalant or food allergens, peripheral blood eosinophilia, and wheezing not related to a cold [8].

Our data revealed that in real life, defining the risk factors associated with the API is quite easy and could be one of the most significant objective indications for prescription systemic GCS for viral-induced wheezing, even in the youngest patients. This is not the case for severity of wheezing, however, because strict and specific criteria defining the level of severity and indications for the introduction of systemic GCS are lacking. Symptoms of a severe and life-threatening asthma attack are usually used for this definition [6]. In addition, the severity of the condition of a child depends on specific viral etiology or even multiple respiratory pathogens. Unfortunately, we did not find studies with evaluated systemic GCS effects on virus-induced wheezing caused by different pathogens.

According to our results, the clinical evaluation of symptom severity in real life could be quite complicated and more subjective rather than objective. We found that only one symptom, increased work of respiratory muscles, represented respiratory distress and was significant in patients treated with GCS. However, if taken alone, it does not always confirm severe wheezing and could lead to both overestimation and overtreatment of wheezing. A better and more strict definition of severe wheezing is needed while describing indications for systemic GCS in conditions other than asthma.

Dexamethasone and prednisolone are the most recommended drugs for the treatment of severe wheezing [1]. According to the literature, oral prednisolone is the steroid of choice for an asthma attack, but there are insufficient data about its safety and efficacy compared with dexamethasone in this situation [6]. Methylprednisolone is typically used because of its sufficient anti-inflammatory properties, low mineralocorticoid, and HPA suppression potency $[13,14]$. Methylprednisolone was the most common systemic GCS used in our study (57.7\%). However, we cannot conclude that methylprednisolone was the drug of choice because of its superiority to other medications. The most possible reason is that most patients received methylprednisolone intravenously and prednisolone was given orally.

Usually, it is recommended to use prednisolone or its equivalent $(1-2 \mathrm{mg} / \mathrm{kg}$ ) for 1 to 5 days $[1,2]$. Our results were consistent with the 
existing recommendations, but there were still 7 patients who were treated with systemic GCS for more than 5 days. Longer duration of treatment was related to longer hospitalization and the lack of treatment efficacy. It is known that genomic mechanisms of GCS show effects after 4 to 24 hours, and non-genomic effects occur even more rapidly [15]. The lack of efficacy after 1 to 5 days treatment leads to a discussion as to whether the GCS treatment was necessary at all.

Therefore, we raise the idea that different indications and treatment options for systemic GCS in acute asthma and other wheezing disorders should be developed. For those who have no asthma, it would be rational to prescribe GCS according to strict indications and to continue administration of the drug only if a positive effect after the first dose is observed.

Our results showed that even a short course of systemic GCS decreased the leukocyte, neutrophil, and eosinophil counts in blood plasma. It is proven that GCS increase leukocytes $[4,16]$ and decrease lymphocytes, eosinophils, and monocytes in blood plasma [4]. Our study did not demonstrate a decrease in lymphocytes or monocytes. On the contrary, we found decreased levels of leukocytes and neutrophils in blood plasma, which were increased in the first blood tests. We predict that such changes could be found due to respiratory infection. In addition, any source of stress, especially the stress caused by illness, increases the white blood cell count and causes neutrophilia [17]. Therefore, the improved condition of a child during treatment could affect our data and cause a rapid decrease in inflammatory cells.

The most common anti-inflammatory effects of GCS include the arrest of proliferation and induction of apoptosis of both T-cells and eosinophils [18]. It is well known that acute asthma is related to exacerbation of airway inflammation, mainly caused by an increased count of eosinophils and their degranulation [19]. Therefore, systemic GCS, which cause accelerated eosinophil death, are strongly recommended during exacerbations in asthmatic patients [9]. Our results also showed that systemic GCS caused the most significant effect on the decrease of eosinophils in blood, but the major reduction was confirmed in allergic children. Most had significantly higher levels of eosinophils from the beginning. In line with other authors, we conclude that the increased count of eosinophils in blood could be one more significant indication for systemic GCS initiation in viral-induced wheezing when a positive effect could be expected [9, 19].
It is well known that systemic GCS can increase a sodium concentration in blood serum: moderate salt-retaining properties are confirmed for prednisolone, and for methylprednisolone, the effect is lower [13]. Despite the fact that methylprednisolone was the most commonly used GCS in our study, the increase in the sodium concentration in the intervention group was still significant. In addition, some cases of hypernatremia and hypokalemia were observed and showed the risk of these effects for GCS users. Salbutamol and intravenous fluids (isotonic sodium chloride solution) were given to all patients, which likely could have affected our data. However, no changes in sodium or potassium concentrations in blood were registered in the control group. These data suggest that all pediatricians should consider the risk of electrolyte changes if systemic GCS are prescribed.

Changes in blood glucose levels were not significant, but there was one case of hyperglycemia $(>11.1 \mathrm{mmol} / \mathrm{L}$ ) in the intervention group after the first day of treatment. More significant cases of hyperglycemia were observed in previous studies [20, 21]. Systemic GCS administration should be considered as a risk factor for children with diabetes with hyperglycemia, even if prescribed as a short course.

The primary results revealed an increased blood cortisol level in 7 children from the intervention group and in 4 children from the control group. Previous studies analyzed the correlation between the severe condition of a child and an increased cortisol concentration [22, 23]. We suspect that our findings in these particular children also represent the severity of symptoms and most likely are associated with the reaction to them. There was no evidence that respiratory infection alone somehow affected the cortisol level.

Dora Liu et al. reported that cortisol suppression is dependent on the dose and duration of GCS, but this relationship is more important for children than adults [24]. The major risk of adrenal suppression in adults occurs after administration of prednisolone $20 \mathrm{mg}$ (or equivalent) for more than 3 weeks [25], while administration of this drug for less than 2 weeks causes only a transient decrease in the cortisol level [26]. These facts could not be assigned for children, because even relatively low doses of GCS are supraphysiological for them [24]. Auron and Raissouni in their review reported that all forms and methods of GCS administration can have a negative impact on the response of the body to stress, causing a lack of physiological increase of the cortisol level during this period [26]. 
We found that the patients treated with GCS demonstrated a significant decrease in the cortisol level already after the first day of treatment. The results obtained on the fourth day showed the same effect: the cortisol level remained decreased for the duration of the study. Moreover, 19.23\% of GCS users had major cortisol suppression, and older children were at greater risk. A previous study by A. Ahmet and colleagues investigated the direct relationship between age and adrenal suppression after a short course of systemic GCS in children, but their data were not statistically significant [27].

According to our results and previous data, many wheezy children in the acute period are prescribed systemic GCS for some days or weeks [1, 8, 9], but side effects of GCS given as short-course treatment are underestimated. Particular recommendations including indications, minimal effective dose, and safe duration for GCS prescription as short-course treatment are needed for the child population. It is very important to clarify all benefits and risks of systemic GCS for patients with diabetes, obese patients, atopic/nonatopic children, and other specific groups.

According to our results, the average stay in the hospital was significantly longer in the intervention group. More severe condition of patients at the beginning of hospitalization might have influenced these findings. However, it may also support the idea that GCS are not effective to treat virus-induced wheezing, especially for non-asthmatic children [9-10, 12].

The main limitation of our study was the small number of patients and a short period of investigation. Moreover, patients received the first dose of systemic GCS at any time of day (i.e., when the physicians decided to prescribe them); therefore, the time of first blood sample varied. All these facts could influence our results. Also, because of the short time of hospitalization, we could not evaluate further changes in blood cortisol levels after the treatment was stopped. The strength of the study is that it showed our needs and limitations in treating wheezy children in real life. We concentrated on a wide range of effects that can be caused by a short-term administration of systemic GCS in children. No previous similar clinical trials were found. Further studies are needed with a more precise selection of study groups and evaluation of both short- and long-term effects of systemic GCS in different groups of wheezy children.

\section{Conclusion}

Clinical signs of atopy and increased work of respiratory muscles, as well as a family history of asthma and recurrence of wheezing, were the major indications for administration of systemic GCS to wheezy children admitted to the hospital. The severity of wheezing was the most subjective and undefined variable, lacking more objective criteria for evaluation. Moreover, GCS for virus-induced wheezing did not reduce the length of stay in the hospital. Systemic GCS, even if prescribed as short-course treatment, mostly reduced the eosinophil count in allergic patients and decreased cortisol levels, especially in older children. Other minor effects on the levels of sodium, leukocytes, and neutrophils could be registered.

Further studies are needed for more precise determination about when to start or discontinue a short course of systemic GCS in wheezy children, especially in conditions different from asthma. The evaluation of risks and benefits of such treatment is of great importance.

\section{Conflicts of interest}

All authors declare no conflicts of interest.

\section{References:}

1. Fernandes RM, Oleszczuk M, Woods CR, et al. The Cochrane Library and safety of systemic corticosteroids for acute respiratory conditions in children: an overview of reviews. Evid Based Child Health. 2014; 9(3): 733-747, doi: 10.1002/ebch.1980, indexed in Pubmed: 25236311.

2. de Benedictis FM, Bush A. Corticosteroids in respiratory diseases in children. Am J Respir Crit Care Med. 2012; 185(1): 12-23, doi: 10.1164/rccm.201107-1174CI, indexed in Pubmed: 21920920.

3. Longui CA. Glucocorticoid therapy: minimizing side effects. J Pediatr (Rio J). 2007; 83(5 Suppl): S163-S177, doi: 10.2223/ JPED.1713, indexed in Pubmed: 18000630.

4. McKay LI, Cidlowski JA. Physiologic and pharmacologic effects of corticosteroids. In: Kufe DE, Pollock RE, Weichselbaum RR et al. (ed.). Holland-Frei Cancer Medicine. 6th edition. Hamilton (ON), BC Decker 2003.

5. Kurukulaaratchy RJ, Fenn M, Twiselton R, et al. The prevalence of asthma and wheezing illnesses amongst 10-year-old schoolchildren. Respir Med. 2002; 96(3): 163-169, indexed in Pubmed: 11905550.

6. British Thoracic Society. British Thoracic Society and Scottish Intercollegiate Guidelines Network. British Guideline On The Management of Asthma. A National Clinical Guide 153. British Thoracic Society London: 107.

7. Foster SJ, Cooper MN, Oosterhof S, et al. Oral prednisolone in preschool children with virus-associated wheeze: a prospective, randomised, double-blind, placebo-controlled trial. Lancet Respir Med. 2018; 6(2): 97-106, doi: 10.1016/S22132600(18)30008-0, indexed in Pubmed: 29373235.

8. Beigelman A, Bacharier LB. Infection-induced wheezing in young children. J Allergy Clin Immunol. 2014; 133(2): 603604, doi: 10.1016/j.jaci.2013.12.001, indexed in Pubmed: 24636478 . 
9. Beigelman A, Chipps BE, Bacharier LB. Update on the utility of corticosteroids in acute pediatric respiratory disorders. Allergy Asthma Proc. 2015; 36(5): 332-338, doi: 10.2500/ aap.2015.36.3865, indexed in Pubmed: 26314814.

10. Panickar J, Lakhanpaul M, Lambert PC, et al. Oral prednisolone for preschool children with acute virus-induced wheezing. N Engl J Med. 2009; 360(4): 329-338, doi: 10.1056/NEJMoa0804897, indexed in Pubmed: 19164186.

11. National Institute for Health and Care Excellence (NICE). Bronchiolitis in children: diagnosis and management. NICE guideline 2015.

12. Collins AD, Beigelman A. An update on the efficacy of oral corticosteroids in the treatment of wheezing episodes in preschool children. Ther Adv Respir Dis. 2014; 8(6): 182-190, doi: 10.1177/1753465814552283, indexed in Pubmed: 25294845.

13. Becker DE. Basic and clinical pharmacology of glucocorticosteroids. Anesth Prog. 2013; 60(1): 25-31; quiz 32, doi 10.2344/0003-3006-60.1.25, indexed in Pubmed: 23506281.

14. Chrousos G, Pavlaki AN, Magiakou MA, et al. Glucocorticoid therapy and adrenal suppression. [Updated 2011 jan 11]. In: de Groot LJ, Dungan K (ed.). . Endotext [Internet]. 2018.

15. Alangari AA. Genomic and non-genomic actions of glucocorticoids in asthma. Ann Thorac Med. 2010; 5(3): 133-139, doi: 10.4103/1817-1737.65040, indexed in Pubmed: 20835306.

16. Average Increases in White Blood Cell (WBC) Counts with Glucocorticoids (e.g., Decamethasone, Methylprednisolone, and Prednisone). [Internet]. Ebmconsult.com. 2015 [cited 30 August, 2017]. Avaiable from: www.ebmconsult.com/articles/ glucocorticoid-wbc-increase-steroids.

17. Riley LK, Rupert J. Evaluation of patients with leukocytosis. Am Fam Physician. 2015; 92(11): 1004-1011, indexed in Pubmed: 26760415.

18. Zimmerman JJ. Adjunctive steroid therapy for treatment of pediatric septic shock. Pediatr Clin North Am. 2017; 64(5): 1133-1146, doi: 10.1016/j.pcl.2017.06.010, indexed in Pubmed: 28941540

19. Kim CK, Callaway Z, Fujisawa T. Infection, eosinophilia and childhood asthma. Asia Pac Allergy. 2012; 2(1): 3-14, doi 10.5415/apallergy.2012.2.1.3, indexed in Pubmed: 22348202.
20. Arkader R, Malbouisson LM, Del Negro GM, et al. Factors associated with hyperglycemia and low insulin levels in children undergoing cardiac surgery with cardiopulmonary bypass who received a single high dose of methylprednisolone. Clinics (Sao Paulo). 2013; 68(1): 85-92, doi: 10.6061/clinics/2013(01) oa14, indexed in Pubmed: 23420163.

21. Wintergerst KA, Foster MB, Sullivan JE, et al. Association of hyperglycemia, glucocorticoids, and insulin use with morbidity and mortality in the pediatric intensive care unit. J Diabetes Sci Technol. 2012; 6(1): 5-14, doi: 10.1177/193229681200600102, indexed in Pubmed: 22401317.

22. Tarjányi Z, Montskó G, Kenyeres P, et al. Free and total cortisol levels are useful prognostic markers in critically ill patients: a prospective observational study. Eur J Endocrinol. 2014; 171(6): 751-759, doi: 10.1530/EJE-14-0576, indexed in Pubmed: 25271243

23. Ballestero Y, López-Herce J, González R, et al. Relationship between hyperglycemia, hormone disturbances, and clinical evolution in severely hyperglycemic post surgery critically ill children: an observational study. BMC Endocr Disord. 2014 14: 25, doi: 10.1186/1472-6823-14-25, indexed in Pubmed: 24628829.

24. Liu D, Ahmet A, Ward L, et al. A practical guide to the monitoring and management of the complications of systemic corticosteroid therapy. Allergy Asthma Clin Immunol. 2013; 9(1): 30, doi: 10.1186/1710-1492-9-30, indexed in Pubmed: 23947590.

25. Guaraldi F, Karamouzis I, Berardelli R, et al. Secondary adrenal insufficiency: where is it hidden and what does it look like? Front Horm Res. 2016; 46: 159-170, doi: 10.1159/000443915, indexed in Pubmed: 27211575.

26. Auron M, Raissouni N. Adrenal insufficiency. Pediatr Rev. 2015; 36(3): 92-102; quiz 103, 129, doi: 10.1542/pir.36-3-92, indexed in Pubmed: 25733761.

27. Ahmet A, Brienza V, Tran A, et al. Frequency and duration of adrenal suppression following glucocorticoid therapy in children with rheumatic diseases. Arthritis Care Res (Hoboken). 2017; 69(8): 1224-1230, doi: 10.1002/acr.23123, indexed in Pubmed: 27723273. 